\title{
MS16-P12 | MoRPhOLOGICAL ANALYSIS OF THE MIGRATION-INDUCED FIELD-STABILIZED POLAR PHASE In SRTIO 3 With SCANNING X-RAY Diffraction Microscopy
}

Weigel, Tina (TU Bergakademie Freiberg, Freiberg, GER); Richter, Carsten (European Synchrotron Radiation Facility (ESRF), Grenoble, FRA); Nentwich, Melanie (TU Bergakademie Freiberg, 09599, GER); Mehner, Erik (TU Bergakademie Freiberg, 09595, GER); Zschornak, Matthias (TU Bergakademie Freiberg, 09599, GER); Meyer, Dirk C. (Tu Bergakademie Freiberg, Freiberg, GER)

Dielectric strontium titanate $\mathrm{SrTiO}_{3}$ (STO) is a perovskite material with space group $\mathrm{Pm}-3 \mathrm{~m}$. During application of an electric field oxygen vacancies migrate to the cathode and oxygen ions to the anode. Slowly, the anode side of the STO crystal transforms into a tetragonal phase, the migration-induced field-stabilized polar (MFP) phase. The MFP phase shows polar properties, as piezoelectricity and pyroelectricity, in contrast to cubic STO. This indicates structural changes due to the electric field. We refined the structure of the MFP, in the same type as $\mathrm{PbTiO}_{3}$ and $\mathrm{BaTiO}_{3}$ with space group $\mathrm{P} 4 \mathrm{~mm}$. The new lattice is a highly strained state of STO, with a strain of $\sim 1 \%$ in field direction. Polar displacements play an important role for the formation of the tetragonal disorder, but the specifics of the forming- and collapsing-process of the polar phase is yet unknown. To gain further insight into these processes, we used Scanning X-ray Diffraction Microscopy to analyse the strain field and morphology of the MFP phase at the Beamline ID01 of the European Synchrotron Radiation Facility. We examined STO crystals in the unformed state, during electroformation with the appearing MFP phase, and after formation. The results will give a better understanding of the thickness dependence of the strain and the metastable character of the polar phase after switching the voltage off. Hopefully, the conclusions are transferable to other strain-driven phase-transitions and their impact on the crystal structure as well as related material properties. 